\title{
Dominant T- and B-cell epitopes in an autoantigen linked to Chagas' disease
}

\author{
Núria Gironès, ${ }^{1}$ Clara I. Rodríguez, ${ }^{1}$ Eugenio Carrasco-Marín, ${ }^{1}$ Reyes Flores Hernáez, ${ }^{2}$ \\ Jacobo López de Rego, ${ }^{2}$ and Manuel Fresno ${ }^{1}$
}

${ }^{1}$ Centro de Biología Molecular, Conrejo Superior de Investigaciones Científicos,
Universidad Autónoma de Madrid, Madrid, Spain
${ }^{2}$ Departamento de Biología Celular, Universidad Complutense de Madrid, Madrid, Spain

Address correspondence to: Manuel Fresno, Centro de Biología Molecular, CSIC-UAM, Universidad Autónoma de Madrid, Cantoblanco, E-28049 Madrid, Spain. Phone: 34-91-3978413; Fax: 34-91-3974799; E-mail: Mfresno@cbm.uam.es.

Received for publication July 11, 2000, and accepted in revised form February 21, 2001.

In Chagas' disease caused by Trypanosoma cruzi, a paradigm of autoimmune disease, both autoantibodies and autoreactive $T$ cells have been described. We have identified a novel dominant autoantigen, named Cha, recognized by the majority of sera from T. cruzi-infected humans and mice. We noted significant homologies between amino acids 120-129 of Cha, where the B-cell epitope maps, and an expressed sequence tag from T. cruzi, and also between amino acids 254-273 of Cha and a repeated amino acid sequence from the shed acute-phase antigen (SAPA) of T. cruzi. Moreover, T. cruzi-infected mice contain autoreactive $T$ cells that can cross-react with Cha and the SAPA homologous peptides. Transfer of $T$ cells from infected mice triggered anti-Cha (120-129) Ab production in naive recipients. Interestingly, heart tissue sections from those adoptive transferred mice showed cardiac pathology similar to T. cruzi-infected mice. Our results demonstrate the presence of both T- and B-cell cross-reactive epitopes in the Cha antigen. This dual mimicry may lead to $\mathrm{T} / \mathrm{B}$ cell cooperation and give rise to a pathological immunodominant response against Cha in $T$. cruzi infected animals.

J. Clin. Invest. 107:985-993 (2001).

\section{Introduction}

Chagas' disease affects several million people in Central and South America and is considered a paradigm of autoimmune disease. It is caused by the protozoan parasite Trypanosoma cruzi, which has three different morphologies: epimastigote, which replicates in the insect vector; trypomastigote, which infects host's cells; and amastigote, which replicates intracellularly in the host's cells (1). Approximately $30 \%$ of infected persons develop symptoms of the disease in their lifetime, which include cardiomyopathy, neuropathies, and dilatation of colon or esophagus (2). The finding of a T-cell rich inflammatory mononuclear cell infiltrate and the scarcity of parasites in heart lesions questioned the direct participation of $T$. cruzi in chronic Chagas' cardiomyopathy (CCC) and suggested the possible involvement of autoimmunity (3), although this remains a hotly debated issue (4).

Autoimmunity can arise from the stimulation of autoreactive $\mathrm{B}$ and/or $\mathrm{T}$ cells in response to foreign antigens originated by the sharing of molecular determinants between a parasite and its host (molecular mimicry) (5). It has been proposed that when there is molecular mimicry between host and parasite, autoimmunity can develop through a T/B-cell cooperation mechanism, but experimental direct evidence is still scarce. Thus, there are many examples of molecular mimicry between microorganisms and host antigens, both at the molecular and antigenic levels. However, molecular mimicry in the context of an infection by organisms bearing the mimicking peptide has been demonstrated in very few cases (6-9).

There are numerous reports of $T$. cruzi antigens cross-reactive with host's heart and neural tissues (10-14), but none of the autoantibodies or autoreactive $T$ cells against those antigens seem to be the leading cause of autoimmune pathogenesis. Recently, Kalil and Cunha-Neto have described myosin as a major antigen of heart-specific autoimmunity and suggested the possible relevance of myosin recognition in human CCC (15). Anti- $\beta$-adrenergic (14) and muscarinic receptor (16) Ab's that cross-react with T. cruzi ribosomal proteins may also cause cardiac pathology. While the presence of "anti-self" immune responses in T. cruzi infections has been unquestionably demonstrated, evidence for the mediation of cross-reactive $\mathrm{Ab}$ 's or $\mathrm{T}$ cells in pathology is still lacking.

Here we report the identification of a host antigen, named Cha, recognized by most chagasic sera. Our results show that reactivity against Cha in human and mice infections is the result of molecular mimicry between clearly distinct Cha $\mathrm{T}$ - and B-cell epitopes and highly immunogenic T. cruzi antigens, which trigger both T- and B-cell responses. Finally, our results suggest that the dominant autoimmune response against Cha is the result of T/B-cell cooperation. This is, to our 
knowledge, the first description of T- and B-cell crossreactive epitopes in the same autoantigen. This dual cross-reactivity leads to the dominant and strong response against Cha during T. cruzi infection that may contribute to pathology.

\section{Methods}

Cloning of Cha cDNA. A 974-bp partial cDNA was isolated by immunoscreening of a human Jurkat cDNA expression library $\lambda$ ZAP Express (Stratagene, La Jolla, California, USA) using a pool of sera from chagasicchronic patients. Full-length Cha cDNA (1,458 bp) was obtained by $5^{\prime}$ rapid amplification of cDNA ends (RACE) using Cha internal-nested oligonucleotides 912$5^{\prime}$-M (5'-GTGGCACCTTCGCCCCATTCTGAAT- $\left.3^{\prime}\right)$ and NGP1 (5'-TGCAAAGCAGTAGTTGTAGCCGCAGT-3'), and the Marathon Kit and Advantage KlenTaq Polymerase Mix (CLONTECH Laboratories Inc., Palo Alto, California, USA). The amplified cDNAs were cloned into pGEM-T vector (Promega Corp., Madison, Wisconsin, USA) and sequenced using the fmol kit (Promega Corp.) or automatically with a Perkin-Elmer Applied Biosystems sequencer (Foster City, California, USA). Nucleic acid and protein sequences were analyzed by the University of Wisconsin Genetics Group Sequence Analysis Software Package.

a

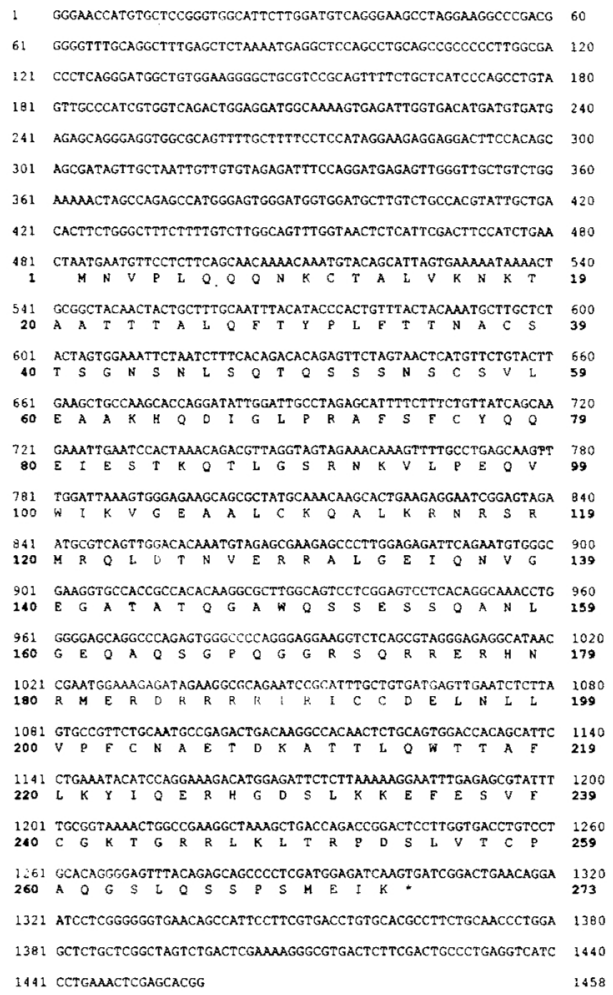

Protein and synthetic peptides. Hen egg-white lysozyme (HEL) was from Sigma Chemical Co. (St. Louis, Missouri, USA) Peptides human R1 (SLVTCPAQGSLQSSPSMEIK), human R3 (MRQLDTNVER), human R3H (MRQLDTNVERRALGEIQNV), mouse R3M (IRQLDTSVERRALGEIQNV), T. cruzi R3T (LRQLDFVEEVLRKHPDKVE), and T. cruzi S1 (STPSTPADSSAHSTPSTPV) were synthesized on an Applied Biosystems Synthesizer Model 431A. A series of ten amino acid (aa) peptides with an overlap of four aa, spanning the entire sequence of short Cha (sCha), were synthesized using a modified multi-pin peptide synthesis (17) that allows the cleavage of peptides from the pins. Peptides were purified by HPLC, and checked for accuracy by mass spectrometry.

$A b$ 's. Human chagasic sera were kindly supplied by S. Gea, D. Iosa, E. Moretti, and B. Basso (Universidad Nacional de Cordoba, Argentina), which included four asymptomatic sera, ten with electrocardiographic alterations, and 26 with electrocardiographic alterations and cardiac failure. Sera from systemic lupus erythematosus (SLE) patients were supplied by J. Sequí (Instituto de Salud Carlos III, Madrid, Spain); sera from human and mice infected with Leishmania infantum were supplied by C. Alonso (Centro de Biología Molecular, Madrid, Spain); and sera from idiopathic dilated cardiomyopathy (IDC) patients were supplied by S. Gea. Polyclonal Ab anti-Cha

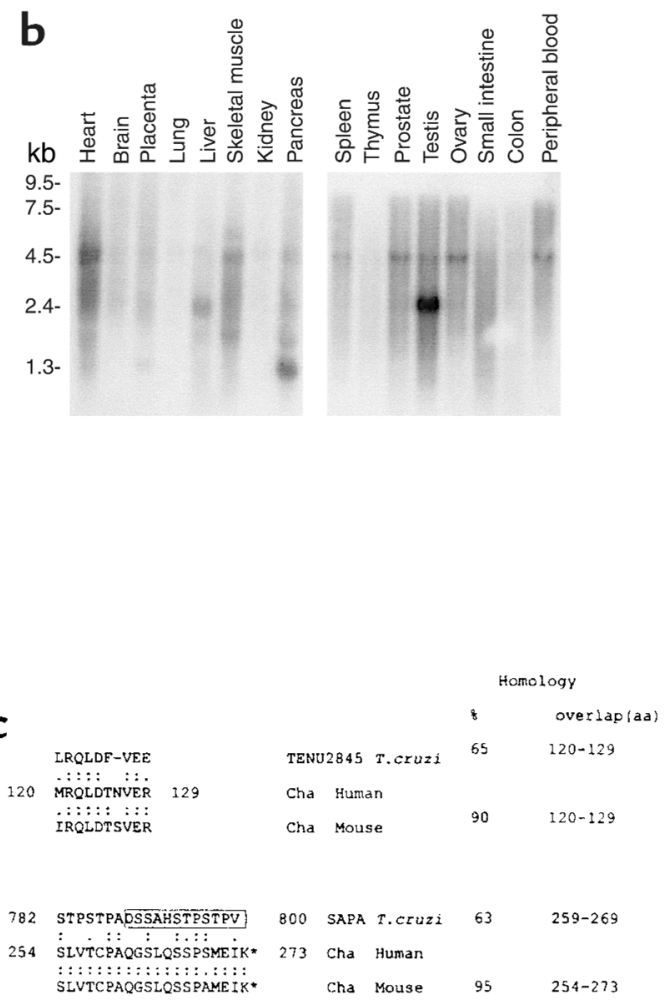

\section{Figure 1}

Expression and sequence of Cha. (a) Nucleotide and deduced aa sequence of Cha. The aa positions are denoted by numbers in boldface; the asterisk indicates the termination codon (TGA). (b) Cha mRNA Northern blot analysis. Nylon membranes containing $2 \mu \mathrm{g}$ of each poly(A) ${ }^{+} \mathrm{RNA}$ from various human tissues were hybridized with a Cha cDNA probe. (c) An aa comparison of human and mouse Cha peptides with T. cruzi antigens. Identical aa's are denoted by two dots and conserved aa by one dot. Percentages of homology are shown. The open box indicates a SAPA COOH-terminal repeat. 
a

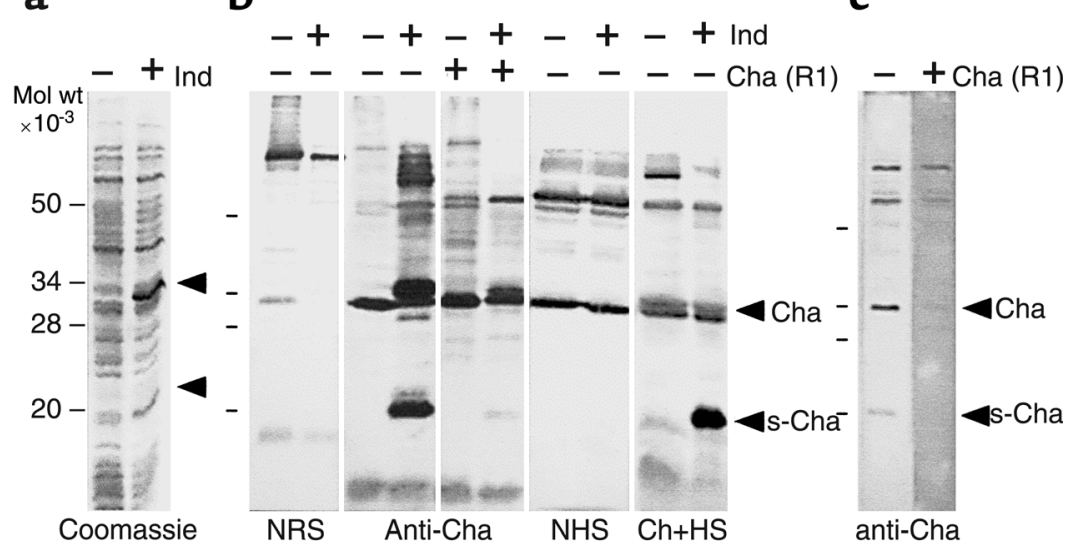

d

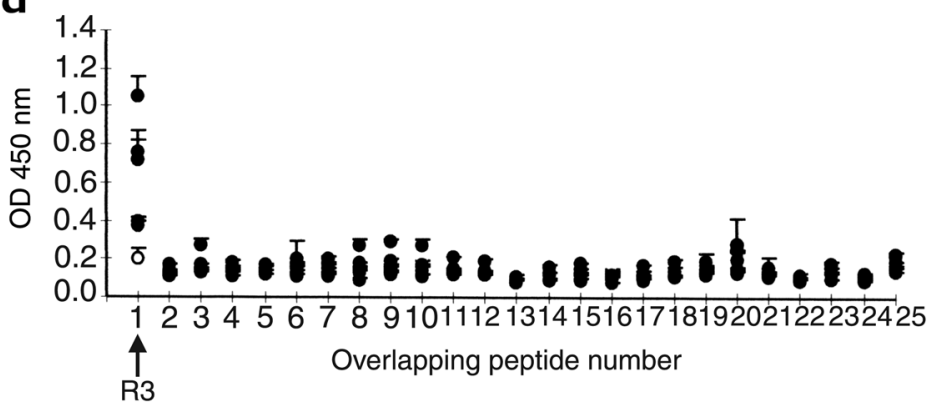

\section{Figure 2}

Reactivity of chagasic sera. (a) Coomassie blue staining shows the expression of recombinant Cha in induced $(+)$ or not induced (-) bacterial lysates of $E$. coli. Arrows indicate Cha and sCha proteins. (b) Western blot analysis of the same lysates with normal rabbit serum (NRS) and anti-Cha and its competition with the immunizing Cha peptide (R1) is shown. The same lysates were tested with normal human serum (NHS) and with chagasic human serum $(\mathrm{Ch}+\mathrm{HS})$. Arrows indicate 34-kDa (Cha) and 20-kDa (sCha) proteins. (c) Western blot analysis of a Jurkat lysate with the anti-Cha $\mathrm{Ab}$. Recognition of specific bands of Cha and sCha is denoted by arrows. (d) Mapping of the sCha epitope recognized by human chagasic sera. The reactivity of six human chronic chagasic sera against 25 overlapping ten-aa peptides spanning sCha was assayed using ELISA. Five sCha-positive sera (filled circles) and one negative sera (open circle) are shown. Peptide 1 corresponds to the R3 sequence. was raised in rabbits against aa $254-273$ of Cha (R1) as described (18). IgG of the preimmune and immune sera obtained after the second boost was purified by ammonium sulphate precipitation. Affinity-purified IgG from human chagasic sera specific for the R3 peptide of Cha was obtained using the SulfoLink Kit (Pierce Chemical Co., Rockford, Illinois, USA).

Bacterial expression of recombinant proteins. A 954-bp insert derived from the original library clone was subcloned into the thermoinducible bacterial expression vector PCYTEX P1 between NdeI and EcoRI sites of the polylinker. This was accomplished by amplifying the fragment using PCR with the original pBKCMV Cha clone with the Cha mut $5^{\prime}$ oligonucleotide (5'GCCTCTTCAGCAACATATGAAATGTACAGCATTA-3'). In addition, a second construct was made to express the protein from a second ATG codon at position 841 of the complete nucleotide sequence, with the oligonucleotide sCha $5^{\prime}$ (5'-AGGAATCGGAGTCATATGCGTCAGTTG-3'). The pCYTEX plasmids were transformed in Escherichia coli strain XL-1Blue and the recombinant proteins prepared as described (19). Recombinant sCha ( $\mathrm{rsCha}$ ) was purified by gel filtration as described (20). Removal of endotoxins from purified rsCha was done with polymyxin B agarose (Sigma Chemical Co.). The presence of endotoxin activity in E-toxate assays (Sigma Chemical Co.) was undetectable.

Immunoassays. For Western blot analysis, Jurkat and J774 cells grown in suspension in complete RPMI-1640 and DMEM (5-10\% FCS), respectively, were centrifuged, and the pellets solubilized with lysis buffer NP-40, pH 7.8 (150
$\mathrm{mM} \mathrm{NaCl}, 1 \% \mathrm{NP}-40,20 \mathrm{mM}$ Tris-HCl, 1 mM PMSF, 1 $\mu \mathrm{g} / \mathrm{ml}$ aprotinin, $1 \mu \mathrm{g} / \mathrm{ml}$ leupeptin, and $1 \mu \mathrm{g} / \mathrm{ml}$ pepstatin) for 30 minutes at $4^{\circ} \mathrm{C}$. Lysates were cleared by ultracentrifugation at $37,100 \mathrm{~g}$ for 15 minutes at $4^{\circ} \mathrm{C}$. Thirty micrograms of total cell lysate were electrophoresed on 13\% SDS-PAGE mini-gels, transferred to Immobilon-P (Millipore, Bedford, Massachusetts, USA) membrane (18) and incubated with anti-Cha at 1:1000 dilution or chagasic sera at 1:100 dilution. Competitions were performed by preincubation of antisera with 100 $\mu \mathrm{g} / \mathrm{ml}$ of peptides for 20 minutes at $22^{\circ} \mathrm{C}$. Then membranes were incubated either with goat anti-rabbit or rabbit anti-human alkaline phosphatase-conjugated (APconjugated) antisera at 1:10,000 dilution (Pierce Chemical Co.). Alternatively, the membranes were incubated with goat anti-human horseradish peroxidase (HRP) and developed with the enhanced chemiluminescence (ECL) reagent (Amersham Pharmacia Biotech, Little Chalfont, United Kingdom),

The specificity of the sera was tested using ELISAs. Recombinant proteins and peptides were incubated overnight at $4^{\circ} \mathrm{C}$ in $50-150 \mu \mathrm{l}$ of $10-100 \mu \mathrm{g} / \mathrm{ml}$. Blocking was done in PBS containing 3\% low-fat dry milk and $0.2 \%$ Tween-20. Sera from chagasic patients and infected mice were used at 1:100 dilutions in PBS, $1 \%$ low-fat dry milk, and $0.05 \%$ Tween- 20 at $4{ }^{\circ} \mathrm{C}$. Wells were washed three times with the same buffer and subsequently incubated with goat anti-human or rabbit anti-mouse HRP-conjugated Ab's for 1 hour at room temperature and washed five times. The positive cutoff for assays was determined from the mean value \pm three SDs of the negative. 
Table 1

Reactivity of human sera against rsCha, R3, R1 peptides of Cha, and S1 peptide of T. cruzi in ELISAs

\begin{tabular}{|c|c|c|c|c|c|c|c|c|c|c|c|c|}
\hline & \multicolumn{3}{|c|}{ rsCha } & \multicolumn{3}{|c|}{ R3 } & \multicolumn{3}{|c|}{$\mathrm{R} 1$} & \multicolumn{3}{|c|}{ S1 } \\
\hline & $n$ & $\%^{A}$ & Mean ${ }^{B}$ & $n$ & $\%$ & Mean & $n$ & $\%$ & Mean & $n$ & $\%$ & Mean \\
\hline Chagasic sera & 40 & 90 & 0.77 & 40 & 97 & 0.95 & 32 & 0 & 0.23 & 32 & 59 & 0.73 \\
\hline IDC & & ND & & 6 & 0 & 0.12 & & ND & & & ND & \\
\hline SLE & 13 & 0 & 0.18 & 13 & 0 & 0.14 & & ND & & & ND & \\
\hline Leishmaniasis & 10 & 0 & 0.22 & 10 & 0 & 0.11 & & ND & & & ND & \\
\hline Normal & 6 & 0 & 0.16 & 6 & 0 & 0.12 & & ND & & & ND & \\
\hline
\end{tabular}

Results are representative of three independent experiments. APercentage of positive sera. ${ }^{B}$ Mean of OD $450 \mathrm{~nm}$. ND, not determined.

Infections and immunizations in mice. Four-week-old female BALB/c, CBA/J, and C57BL/6 mice were infected with different strains (Tulahuen and MC) of T. cruzi by intraperitoneal injection of $10^{4}$ trypomastigotes (indicated if different) from blood of a previously $T$. cruzi-infected mouse. Mice were bled at different days after infection, and serum was obtained. Parasitemia was measured as described (21). CBA/J and C57BL/6 mice were immunized in the rear footpads with $10 \mathrm{nmol}$ of rsCha, Cha peptides, and HEL emulsified in CFA (Sigma Chemical Co.). Spleens from infected mice and popliteal lymph nodes from immunized mice were elicited, and cells were obtained using a cell strainer (Becton Dickinson Labware, Franklin Lakes, New Jersey, USA). After lysing red blood cells with water for 5 seconds, the cells were washed twice in DMEM and resuspended in DMEM $10 \%$ FCS (DMEM10). Cells were plated in 96-well plates $\left(2 \times 10^{5} / 200 \mu \mathrm{l}\right)$ containing $5 \mu \mathrm{g} / \mathrm{ml}$ of ConA or with antigens $(0.5$ to $500 \mu \mathrm{g} / \mathrm{ml})$, as indicated. Proliferation was measured by incorporation of $1 \mu \mathrm{Ci}\left[{ }^{3} \mathrm{H}\right]$ thymidine (Amersham Pharmacia Biotech) per well during the last 24 hours of a 3-day culture for spleen cells or of a 4-day culture for lymph node cells. Cells were then harvested on a glass-fiber filter using a Cell Harvester (Skatron Instru- ments Inc., Sterling, Virginia, USA), and radioactivity was estimated. Results are expressed as relative proliferation index $\left[P=(\mathrm{cpm} \text { with antigen } / \mathrm{cpm} \text { with medium })_{\text {infected }}-\right.$ (cpm with antigen/cpm with medium $)_{\text {uninfected }}$. T. cruzi epimastigotes, blood trypomastigotes, and amastigotes were obtained as described (22).

Adoptive transfer. CBA/J spleen cells from mice infected with $5 \times 10^{3}$ Tulahuen trypomastigotes were obtained at 3 months after infection as described previously. B cells and macrophages were depleted by incubation with 20 $\mu \mathrm{g}$ of $40 \mathrm{~F} \mathrm{Ab}\left(\right.$ anti-IA $\left.^{\mathrm{k}}\right), 20 \mu \mathrm{g}$ anti-mouse IgM, $20 \mu \mathrm{g}$ anti-mouse IgG, plus guinea pig complement in DMEM10 for 2 hours. Cells were washed twice in DMEM, resuspended at $10^{7}$ cells $/ \mathrm{ml}$ in DMEM10, and incubated for 2 hours in a nylon wood column at $37^{\circ} \mathrm{C}$. Cells were eluted with prewarmed DMEM 10 at $37^{\circ} \mathrm{C}$. Up to $99 \%$ of the cells were CD3 positive. T cells $\left(10^{7}\right)$ were injected intraperitoneally into naive CBA/J mice.

Histological staining. Mouse cardiac tissue was fixed in $10 \%$ formol for 72 hours at room temperature, rinsed, and transferred into $70 \%$ ethanol. Pieces were dehydrated in ethanol of growing gradation, and benzol, embedded in paraffin, and cut into sections from 8 to $10 \mu \mathrm{m}$. Tinctures used were hematoxylin and eosin (23)
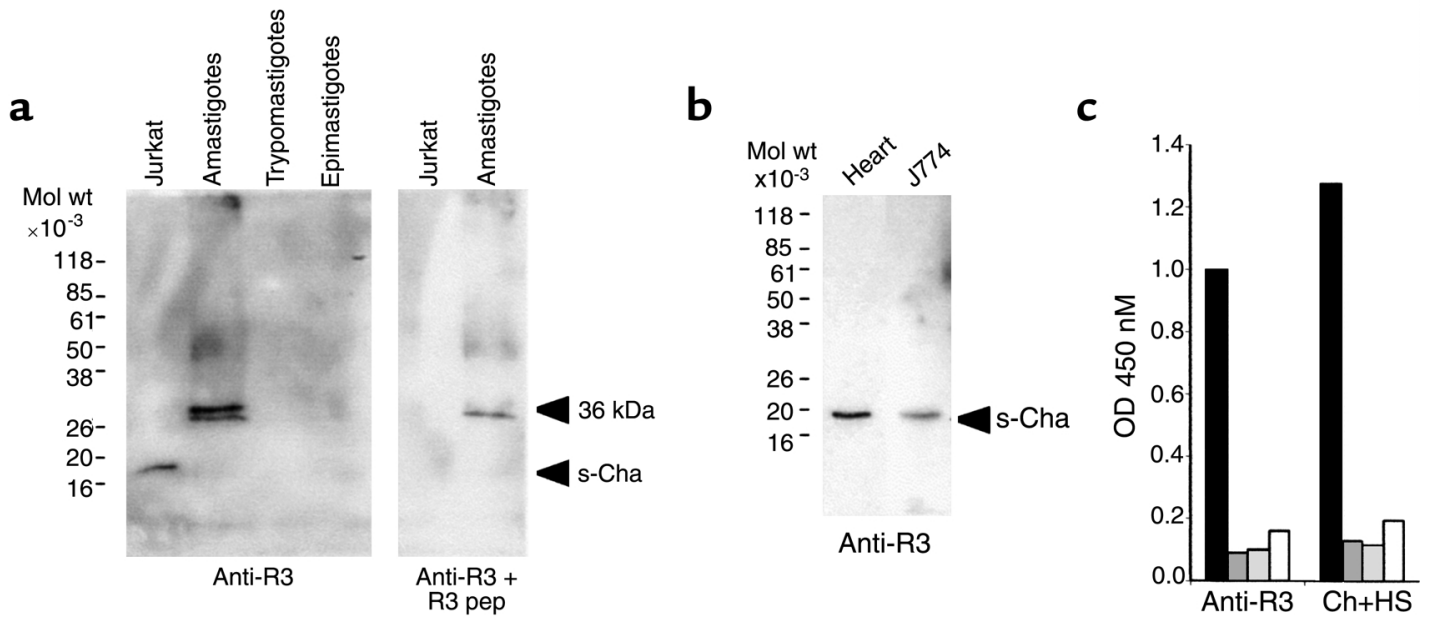

Figure 3

Reactivity of human anti-R3 Ab's. Western blot analysis with affinity-purified anti-R3 Ab's from human chagasic sera. (a) Reactivity against sCha $(20 \mathrm{kDa})$ antigen in Jurkat cells and a $36-\mathrm{kDa}$ antigen present in T. cruzi amastigotes is shown. (b) sCha is expressed in mouse heart and the mouse macrophage $\mathrm{J} 774$ cell line. (c) Competitive R3 peptide ELISAs show that R3 homologous peptides from human (dark gray boxes), mice (light gray boxes), and T. cruzi (open boxes) competed out the binding of either affinity-purified anti-R3 Ab's or whole sera $(\mathrm{Ch}+\mathrm{HS})$ from human chagasic patients to the human R3 peptide (filled boxes). 
and van Gieson's stain (24). The series of pieces were studied using a Leitz-Dialux microscope, equipped with a WILD MPS 51S camera with Photoautomat MPS-4S (Leica, Wetzlan, Germany).

\section{Results}

Identification of a novel, dominant chagasic autoantigen, Cha. In a search for dominant autoantigens in Chagas' disease, we found a human cDNA clone (Cha) (Genbank accession number AJ271337) recognized by most of the chronic chagasic sera (Figure 1a). The Cha gene has $84 \%$ to $89 \%$ identity with several expressed sequence tags (ESTs) from mice (AI595097, AA061706, AI595248, AI428028, MMTEST342, AI427864, and AA061826), which spanned most of the codifying sequence of the human gene, indicating the existence of a Cha orthologue in mouse.

Northern blot analyses showed an ubiquitous expression of Cha mRNA, which is a species of $2.4 \mathrm{~kb}$ and very abundant in testis (Figure 1b). Additionally, a 5-kb mRNA was observed in heart, skeletal muscle, spleen, prostate, ovary, and peripheral blood, and a $1-\mathrm{kb}$ mRNA was observed in the pancreas. No bands were detected in brain, placenta, lung, kidney, thymus, and colon.

There was $65 \%$ homology in a ten-aa overlap between aa 120-129 of Cha, named R3, and the putative gene product of TENU2845 of T. cruzi (Figure 1c), and 63\% homology in a 11-aa overlap (259-269) between aa 254272 of Cha, named R1, and the COOH-terminal nonapeptide repeats of the shed acute-phase antigen (SAPA) of T. cruzi, named S1 (Figure 1c). Those percentages of homology agree with the Weise and Carnegie criteria for mimicry-type similarities between peptides (25).

Reactivity of chagasic sera against Cha. To study the crossreactivity of Cha at the molecular level, recombinant Cha was prepared by subcloning its cDNA in the thermoinducible bacterial expression vector PCYTEXP1 (20) and expressing it in E. coli. We observed the specific induction of a protein of the expected size $(34 \mathrm{kDa})$, as well as an additional protein of about $20 \mathrm{kDa}$, named sCha (Figure $2 \mathrm{a})$. The sCha was the result of a secondary translation product from an initiation codon present at position 841 of the nucleotide sequence and lacks aa 1-119 of Cha (see Figure 1). The anti-Cha antiserum recognized not only a $34-\mathrm{kDa}$ protein but also the $20-\mathrm{kDa}$ protein (Figure $2 \mathrm{~b}$ ). Surprisingly, all chagasic sera tested recognized mainly sCha (one of them shown in Figure 2b).

Next, we studied the expression of the Cha antigen in human cells using Western blot analysis. Figure $2 \mathrm{c}$ shows that anti-Cha antiserum recognized 80-, 60-, 34-, and 20$\mathrm{kDa}$ proteins. Only the $34-$ and $20-\mathrm{kDa}$ proteins, corresponding to the predicted sizes of Cha and sCha, respectively, appeared to be recognized specifically, since they were competed out by the immunizing R1 peptide. Similar results were found in other human and mouse cell lines derived from different tissues (not shown).

ELISAs showed very high autoantibody titers against purified recombinant rsCha in a high percentage of
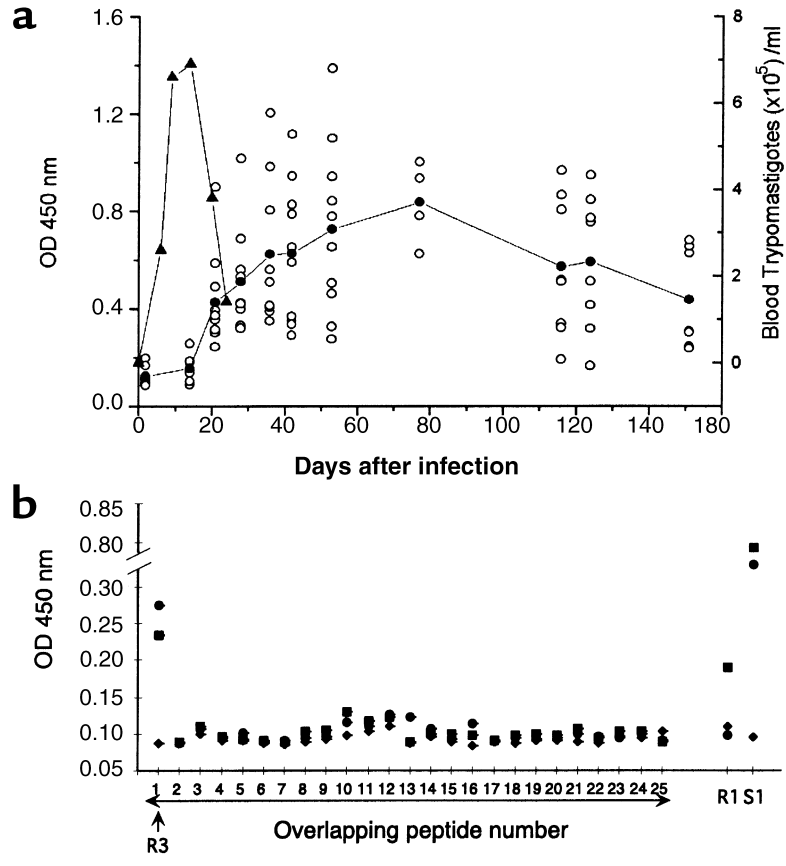

\section{Figure 4}

Cha autoantibodies in experimental $T$ cruzi infections. (a) Reactivity against $\mathrm{rsCh}$ of sera from five mice infected with the Tulahuen and five infected with the MC strains of T. cruzi at different days after infection. Seen are values of individual mice (open circles) and mean of OD values of all mice (filled circles). Parasitemia values are expressed as trypomastigotes $\left(10^{-5} / \mathrm{ml}\right)$ (filled triangles). (b) Mapping of the sCha epitope recognized by infected mice sera. $T$. cruzi-infected mice sera against ten-aa overlapping peptides spanning sCha were assayed. Reactivity of uninfected sera (filled diamonds) 21 days after infection (filled squares) and 53 days after infection (filled circles). Reactivity of the same sera against the S1 peptide of T. cruzi and R1 peptide of Cha is seen.

chagasic sera from different endemic areas of Latin America (90\%). No significant titers against rsCha were observed in sera from healthy individuals from the same area, autoimmune systemic lupus erythematosus (SLE) patients, or patients infected with Leishmania, a $T$. cruzi-related parasite (Table 1).

Molecular characterization of T. cruzi/Cha cross-reactivity. To map the Cha epitope recognized by chagasic sera, we synthesized 25 overlapping peptides spanning sCha and used them in ELISAs of chagasic sera. Interestingly, five out of six chronic chagasic sera tested (Figure 2d) reacted exclusively with a unique peptide, which corresponds to aa 120-129 of Cha, named R3. More interestingly, $97 \%$ of chronic chagasic sera were highly reactive with R3 in comparison with the absence of reactivity in sera from SLE, from patients infected with Leishmania, or suffering IDC, a disease with cardiac symptoms similar to Chagas' disease (Table 1). While $59 \%$ of chronic chagasic sera contained Ab's against the T. cruzi peptide S1, reactivity against the $63 \%$ homologous Cha R1 peptide (aa 254-273) was almost undetectable (Table 1), indicating that infection-induced anti-S1 Ab's did not cross-react with the R1 peptide. 
a

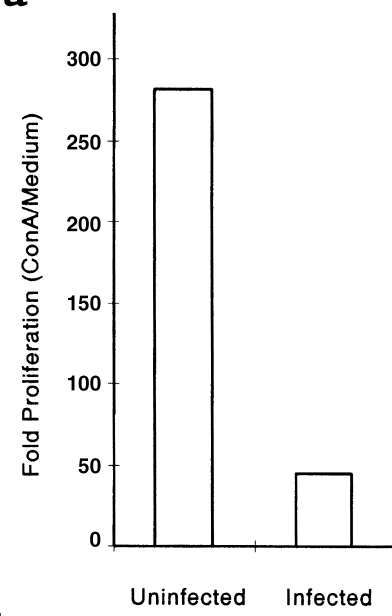

$\downarrow$

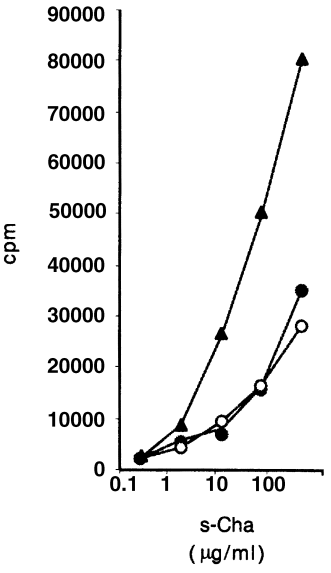

b

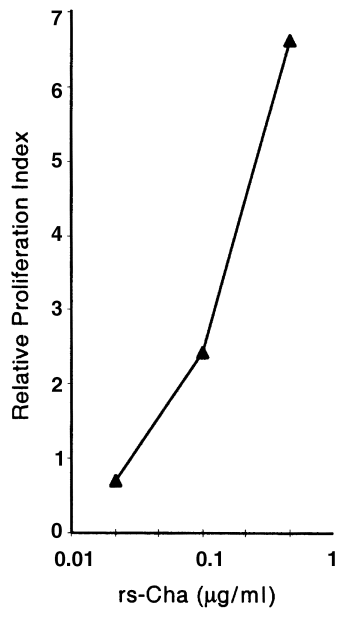

e

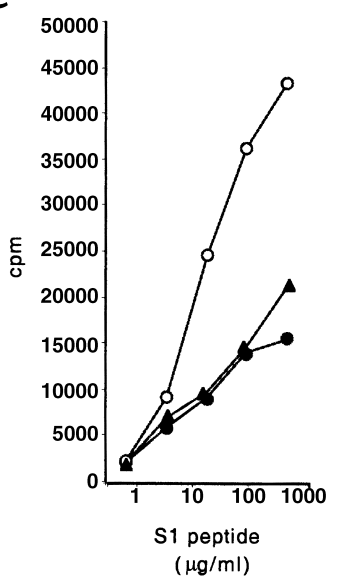

C

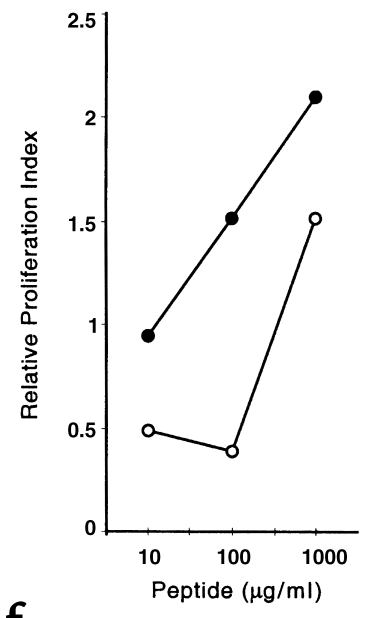

f

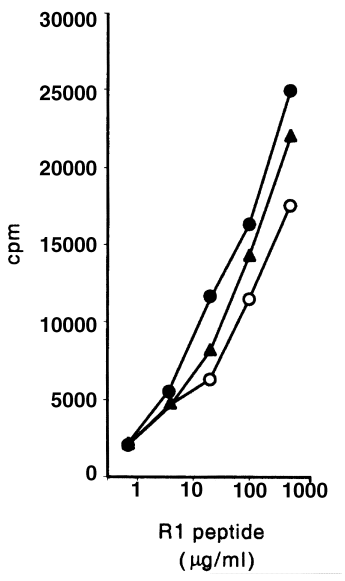

Figure 5

Proliferative response to $\mathrm{rsCha}$ in infected and immunized CBA/J mice. (a-c) Mice were either uninfected or infected with T. cruzi. Spleen cells were cultured in triplicate with ConA, rsCha, R1, and S1 peptides. (a) Fold proliferation of ConA-treated cultures normalized with respect to medium alone in uninfected and 14 days after infection is seen. (b) The rsCha dose-response proliferation (filled triangles) of spleen cells from infected mice and (c) dose-response proliferation to the $\mathrm{S} 1$ (open circles) and R1 (filled circles) peptides of spleen cells from infected mice are seen. The relative proliferation index was calculated as indicated in Methods from the cpm observed in two uninfected and two T. cruzi-infected mice. (d-f) Mice were immunized with rsCha (filled triangles), S1 (open circles), or R1 (filled circles) and their $T$ cells were assayed for proliferation in the presence of different peptides: (d) rsCha, (e) S1, and (f) R1 at indicated concentrations. Results are representative of three independent experiments.
As with recombinant proteins, human chagasic antiR3 purified Ab's preferentially recognized sCha in Jurkat cells (Figure 3a). In addition, they reacted with a $36-\mathrm{kDa} T$. cruzi antigen that is expressed only in amastigotes that may represent the antigen coded by TENU2845. The recognition of both host and parasite antigens was specific since it was competed with the R3 peptide. We also detected sCha in mouse heart extract and the mouse macrophage cell line J774 (Figure 3b), in agreement with the ubiquitous expression of Cha mRNA. Moreover, homologous R3 peptides from humans, mice, and T. cruzi were cross-reactive since anti$\mathrm{R} 3$ purified $\mathrm{Ab}$ binding to the human $\mathrm{R} 3$ peptide $(\mathrm{R} 3 \mathrm{H})$ was competed out with both mouse and T. cruzi peptides (R3M and R3T, respectively) in ELISAs (Figure 3c).

Kinetics of autoantibody formation against Cha in infected mice. We analyzed experimental infections in mice in order to obtain information about the mechanism of autoantibody formation. As in humans, there was a strong autoantibody response against rsCha in all $\mathrm{BALB} / \mathrm{c}$ mice infected with two different strains of $T$. cruzi mice (Tulahuen and MC). Ab's against rsCha began to be detected 21 days after infection, peaking at 77 days after infection and decreasing thereafter, but still being detectable at 153 days after infection, the latest time point analyzed (Figure 4a). The autoantibody response was specific for T. cruzi infection since we did not detect reactivity against rsCha in sera isolated from mice infected with the related parasite L. infantum (not shown).

The corresponding R3 peptide from mouse sCha antigen deduced from the reported nucleotide sequence of several ESTs was $90 \%$ homologous to human R3 (Figure 1c). Thus, we studied whether mouse R3 was also recognized by sera from infected mice. Autoantibody response in $\mathrm{BALB} / \mathrm{c}$ mice during $T$. cruzi infection was also directed against R3 of Cha (Figure $4 \mathrm{~b}$ ). As in humans, despite the appearance of anti$\mathrm{S} 1 \mathrm{Ab}$ 's as infection progressed, no anti-R1 reactivity could be detected (Figure 4b). Similar results were found in infected CBA/J mice strain (not shown).

Identification of a T-cell epitope of Cha cross-reactive with SAPA of T. cruzi. To ascertain whether Cha could also trigger autoreactive $T$ cells during $T$. cruzi infection, spleen cells from CBA/J infected mice (14 days after infection) were assayed for proliferation in the presence rsCha. Proliferation to ConA of spleen cells from infected mice (50fold) was strongly suppressed in comparison with uninfected mice (280-fold) (Figure 5a), as described previously (26). Despite this, rsCha induced a greater than sixfold increase in proliferation in spleens from 
infected mice in comparison with uninfected mice (Figure 5b). Interestingly, the S1 peptide and its homologous peptide R1 were able to induce significant proliferation in infected mice (Figure 5c). To further characterize the $\mathrm{T}$-cell response against Cha, mice were immunized with the Cha antigen as well as the R1 and S1 peptides. The results showed that $\mathrm{T}$ cells isolated from mice immunized with the $S 1$ peptide proliferated in the presence of the $S 1$ peptide and additionally in the presence of rsCha and R1 peptide. Also, T cells isolated from mice immunized with rsCha proliferated in the presence of rsCha and additionally in the presence of the R1 and S1 peptides. Finally $\mathrm{T}$ cells isolated from mice immunized with the R1 peptide of Cha proliferated in the presence of R1 and additionally in the presence of rsCha and S1 peptide (Figure 5, d-f). Interestingly, spleen cells from nonsusceptible C57BL/6 mice immunized with $\mathrm{S} 1$ required 200 -fold higher dose than from susceptible mice to induce a similar level of proliferation (not shown). Proliferation of HEL-specific $\mathrm{T}$ cells to different peptides was not significant (not shown).

Production of anti-R3 Ab's in T. cruzi-susceptible, nonsusceptible, and adoptive transferred mice. It has been described that both T. cruzi-susceptible and nonsusceptible mouse strains develop autoantibodies during T. cruzi infection, but only the first ones developed a strong cardiac pathology (27). Therefore, we studied whether anti- $\mathrm{R} 3 \mathrm{Ab}$ production was different in susceptible and nonsusceptible mouse strains. Despite similar anti-T. cruzi IgG titers in the sera of all strains of infected mice (Figure 6a), antiR3 titers were much lower in the nonsusceptible mouse strain $(\mathrm{C} 57 \mathrm{BL} / 6)$ than in susceptible mice strains $(\mathrm{BALB} / \mathrm{c}$ and $\mathrm{CBA} / \mathrm{J}$ ) (Figure $6 \mathrm{a})$. On the other hand, adoptive transfer of $\mathrm{T}$ cells from chronically infected mice promoted the production of anti-R3 Ab's in recipients at 60 days after transfer (Figure 6b). These anti-R3 $A b$ 's recognized equally well the $\mathrm{R} 3$ peptides from human, mice, and T. cruzi antigens.

Histological analyses of cardiac tissue from T. cruzi-infected and adoptive transferred mice. To assess the possible pathogenic role of $\mathrm{T}$ cells from infected mice we studied whether recipient mice developed pathology. Signs of inflammation in dispersed areas of the myocardium with mild edema, rise in collagen fibers, as well as macrophage and T-cell infiltrates were observed in CBA/J infected mice (Figure 7, a-d). The lymphocytes appeared sometimes thickly gathered among myocytes and with higher frequency in the neighboring endocardium. At the endocardiac level, the regularity of the epithelium was altered; in some areas, little collagen fiber bundles perpendicular to the free surface are seen. Cells with big nuclei of thin chromatin and light eosinophilic cytoplasm were found near some others with clear macrophage-like aspect, as well as a great number of lymphocytes. These results, which are compatible with cardiopathy, were accompanied by erythrocyte fixation in the contact area with ventricular lumen. Interestingly, myocardium of mice transferred with $T$ cells from $T$. cruzi-infected mice (Figure 7, $\mathrm{e}-\mathrm{h}$ ) showed damages similar to T. cruzi-infected mice, although somewhat less exacerbated. Similar effects were seen in the BALB/c strain (not shown).

\section{Discussion}

We have identified here a novel human and mice autoantigen named Cha. A short form of Cha (sCha), present in leukocytes and heart, among other cell types, was recognized by most human chagasic and all chronically infected mice sera regardless of the infecting T. cruzi strain, showing that $\mathrm{Cha}$ is a dominant autoantigen during T. cruzi infection. Cha has two regions of homology, R1 and R3, with two different T. cruzi proteins, SAPA and TENU2845, respectively. This dual homology of Cha with $T$. cruzi strongly suggested that molecular mimicry was the origin of the dominant autoreactivity against Cha observed during infection in both humans and mice. Interestingly, this molecule was recognized by $\mathrm{T}$ and $\mathrm{B}$ cells at different epitopes. Thus, B cells recognized the R3 epitope. On the contrary, T cells specific for S1 of SAPA generated in infected mice recognized R1. In addition,

a
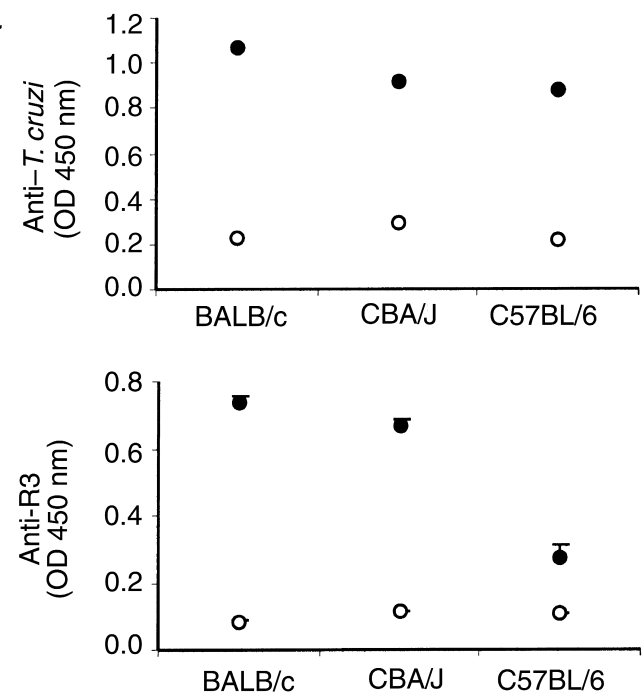

b

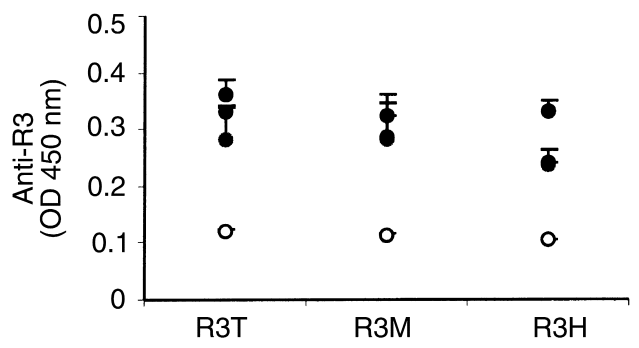

Figure 6

Reactivity against T. cruzi and the R3 peptide in sera from different $T$. cruzi-infected mice strains and adoptive transferred mice. (a) Sera from uninfected (open circles) or chronically infected (filled circles) $\mathrm{BALB} / \mathrm{c}, \mathrm{CBA} / \mathrm{J}$, and $\mathrm{C} 57 \mathrm{BL} / 6$ mice were tested against a T. cruzi lysate and a R3 peptide using ELISAs. (b) Sera from adoptive CBA/J transferred mice was tested by ELISA with peptides from $T$. cruzi (R3T), mice (R3M), and humans (R3H) 2 months after transfer. Results are representative of three independent experiments. 

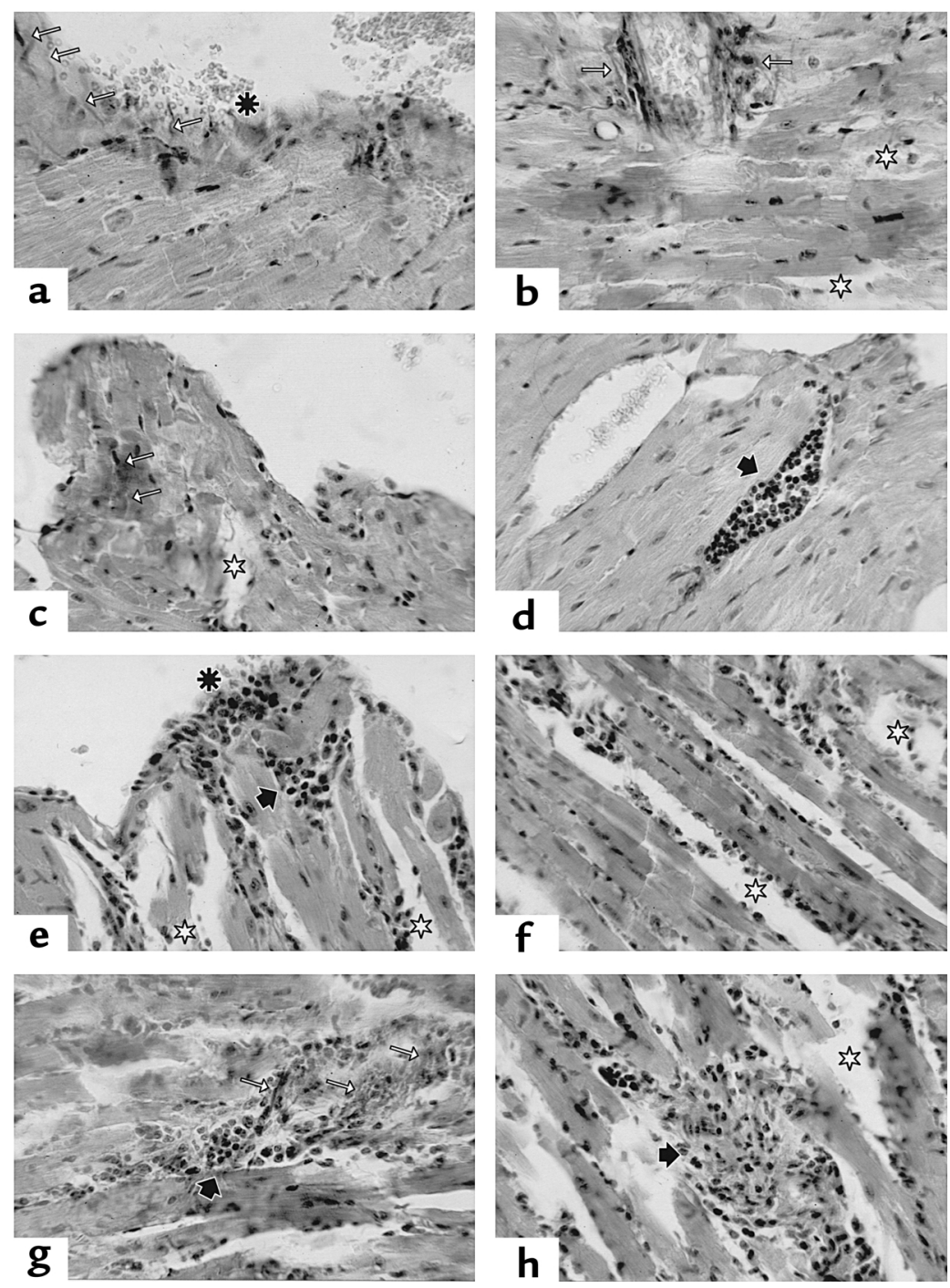

\section{Figure 7}

Histologic study of heart tissue sections from $T$. cruzi and adoptive transferred mice. (a-d) $\mathrm{CBA} / \mathrm{J}$ mice in chronic phase of $T$. cruzi infection. (a) endocarditis (asterisk); note the increase of collagen fibers perpendicular to the tissue surface (white arrows); (b and $\mathbf{c}$ ) strong presence of perivascular and intermyofibrillar collagen fibers (white arrows); disperse area of edema (white stars); (d) vascular dilatation with lymphocytic thrombus (black arrows). (e-h) Cardiac tissue section of CBA/J mice 2 months after transfer of $T$ cells from chronically infected CBA/J mice, as above. (e) limited myocarditis zone (asterisk) with subendocardiac lymphocytic cumulus (black arrow): (e, f, and $\mathbf{h}$ ) general edema (white stars); (g) persistence of collagen fibers (white arrow) with strong lymphocytic cumulus in the myocardium (black arrow). van Gieson staining was done in all the sections except for sections $\mathbf{e}$ and $\mathbf{h}$, where a hematoxylin and eosin staining was performed. $\times 400$.

with $\mathrm{S} 1$ proliferate to rsCha and $\mathrm{R} 1$ peptide and vice versa. These results showed that $\mathrm{T}$ cells specific for $\mathrm{S} 1$ were able to recognize the $\mathrm{R} 1$ peptide in antigen-presenting cells. These S1/R1 autoreactive $\mathrm{T}$ cells present in normal mice could be triggered easily by the high concentration of SAPA, the predominant antigen released during the acute phase to the bloodstream of infected mice (29).

Both molecular mimicry and bystander damage have been proposed transfer of $\mathrm{T}$ cells from infected mice induced anti-R $3 \mathrm{Ab}$ production in recipients. This result demonstrated that anti- $\mathrm{R} 3 \mathrm{Ab}$ production was T-cell dependent and favored the molecular mimicry hypothesis. Although a linked Tand B-cell recognition had been described previously in autoimmune arthritis (9), we now describe, we believe for the first time, cross-reactive T- and B-cell epitopes in the same molecule and in the context of an infection.

The COOH-terminal nonapeptide repeats of SAPA of T. cruzi have been described as highly immunogenic, being most of the $\mathrm{Ab}$ response against the parasite restricted to this domain during the acute phase of infection with T. cruzi (28). Although we observed the presence of anti-S1 Ab's in human and mice infections, they did not bind to the R1 peptide of Cha. This result is somewhat surprising since the titer of Ab's against $\mathrm{S} 1$ is high and R1 and S1 peptides have significant homology at the molecular level. In contrast, spleen $\mathrm{T}$ cells from infected mice were able to proliferate to Cha and to both $\mathrm{S} 1$ and $\mathrm{R} 1$ peptides, despite the strong immunosuppression observed during the acute phase of infection. $\mathrm{T}$ cells isolated from mice immunized as possible mechanisms to activate autoreactive $\mathrm{T}$ or $\mathrm{B}$ cells $(5,30,31)$. Since Cha is expressed in cardiac tissue as well as macrophages, and these cells are destroyed in IDC and leishmaniasis, respectively, in a extent similar to Chagas' disease, our results strongly indicate that tissue damage is not enough to drive the autoimmune response against $\mathrm{Cha}$. Taken together our results suggest that anti-R3 Ab's are not induced by the autoantigen but by the parasite. Nevertheless, once $T$ cells are activated, the presence of the parasite is not required to produce anti-R3 Ab's, as seen in transfer experiments of $\mathrm{T}$ cells from chronically infected mice. In addition, our results revealed the existence of a T. cruzi 36-kDa antigen recognized by anti-R3 Ab's.

So far, some autoantigens have been identified in Chagas' disease and to be related to pathology, such as the $\beta 1$ adrenergic receptor, a 48-kDa host antigen, and cardiac myosin; however, none of these autoantigens seem to be recognized by such a large number of chagasic sera as Cha. Thus, high titer of Ab's against R3 were detected in $97 \%$ of chronic chagasic sera from different and broadly separated endemic areas, but not in sera from healthy individuals or from patients suffering leishmaniasis, a 
related parasitic infection, or other autoimmune diseases, or from IDC, a disease with cardiac symptoms similar to CCC. Moreover, all susceptible infected mice strains, regardless of the infecting strain of T. cruzi, produced Ab's against $\mathrm{R} 3$ of $\mathrm{Cha}$, while nonsusceptible mice infected with $T$. cruzi and mice infected with the related parasite Leishmania infantum did not. Those results indicated that anti-R3 Ab's are very specific to T. cruzi infection.

It has been described that nonsusceptible infected mice do not develop cardiac pathology (27); here we show that they did produce much lower levels of antiR3 Ab's than susceptible mice, and their T cells required 200 -fold more S1/R1 to proliferate than susceptible mice. We think that the low T-cell response against the SAPA peptide is the origin of the decrease in anti-R3 $\mathrm{Ab}$ production. Besides, adoptive transfer of $\mathrm{T}$ cells from infected mice induces anti-R3 Ab's and pathology in the transferred mice. More interestingly, these recipient mice showed signs of heart inflammation, thus the autoimmune response against Cha might be pathogenic. Thus, anti-R3 Ab's are not only very specific to T. cruzi infection, but also related to pathogenesis. Taken together, these results suggest a pathogenic role for the autoimmune response against Cha.

In summary, we have identified a novel autoantigen in Chagas' disease with two defined epitopes, R1 and R3 ( $T$ and $\mathrm{B}$, respectively), that mimic two different $T$. cruzi antigens (SAPA and 36-kDa/TENU2845). This dual mimicry and the $\mathrm{T} / \mathrm{B}$-cell cooperation triggered the dominant response against Cha during T. cruzi infection and may lead to pathology. To our knowledge, this is the first report describing that the transfer of $\mathrm{T}$ cells from $T$. cruzi-infected mice also transfers cardiac pathology. Furthermore, it also unequivocally shows that pathology can be induced in the heart in the absence of infiltrating parasites, contrary to what has been suggested (32). Future experiments will focus on the isolation of the 36$\mathrm{kDa} / \mathrm{TENU} 2845$ T. cruzi antigen and the study of the mechanism by which autoantibodies and/or autoreactive $T$ cells specific for Cha may cause damage in target tissues.

\section{Acknowledgments}

This work was supported from Ministerio de Educación y Cultura, Fondo de Investigaciones Sanitarias, Comunidad Autónoma de Madrid, and Fundación Ramón Areces. We thank Maria A. Chorro and Josefa González for their technical assistance. N. Gironès was recipient of a Comunidad Autónoma de Madrid fellowship. E. Carrasco-Marin was recipient of a Ministerio de Educación y Cultura fellowship.

1. Brener, Z. 1973. Biology of Trypanosoma cruzi. Annu. Rev. Microbiol. 27:347-382.

2. 1991. Control of Chagas' disease. World Health Organization. Technical Report Series, No. 811. Geneva, Switzerland. 10 pp.

3. Schmuñis, G.A. 1987. Autoimmunity in Chagas' disease. Mem. Inst. Oswaldo Cruz. 82:287-310.

4. Kierszenbaum, F. 1996. Chronic chagasic tissue lesions in the absence of Trypanosoma cruzi: a proposed mechanism. Parasitol. Today. 12:414-415.

5. Oldstone, M.B.A. 1989. Molecular mimicry as a mechanism for the cause and as a probe uncovering etiologic agent(s) of autoimmune disease.
Curr. Top. Microbiol. Immunol. 145:128-135.

6. Wucherpfenning, K.W., and Strominger, J.L. 1995. Molecular mimicry in T-cell mediated autoimmunity: viral peptides activate human $\mathrm{T}$ cell clones specific for myelin basic protein. Cell. 80:695-705.

7. Zhao, Z., Granucci, F., Yeh, L., Schaffer, P., and Cantor, H. 1998. Molecular mimicry by herpes simplex virus-type 1: autoimmune disease after viral infection. Science. 279:1344-1347.

8. Gross, D.M., et al. 1998. Identification of LFA-1 as a candidate autoantigen in treatment-resistant Lyme arthritis. Science. 281:703-706.

9. Matsumoto, I., Staub, A., Benoist, C., and Mathis, D. 1999. Arthritis provoked by linked T and B cell recognition of a glycolytic enzyme. Science. 286:1732-1735.

10. Wood, J.N., Hudson, L., Jessell, T.M., and Yamamoto, M. 1982. A monoclonal antibody defining antigenic determinants on subpopulations of human neurons and Trypanosoma cruzi parasites. Nature. 296:34-38.

11. Petry, K., and Eisen, H. 1989. Chagas disease: a model for the study of autoimmune diseases. Parasitol. Today. 5:111-116.

12. Van Voorhis, W., Schelekewy, L., and Trong, H. 1991. Molecular mimicry by Trypanosoma cruzi: the FL-160 epitope that mimics mammalian nerve can be mapped to a 12-amino acid peptide. Proc. Natl. Acad. Sci. USA. 88:5993-5997.

13. Bonfá, E., Viana, V.S.T., Barretto, A.C.P., Yoshinari, N.H., and Cossermelli, W. 1993. Autoantibodies in Chagas' disease: an antibody crossreactive with human and Trypanosoma cruzi ribosomal proteins. $J$. Immunol. 150:3917-3923.

14. Ferrari, I., et al. 1995. Molecular mimicry between the immuno-dominant ribosomal protein $\mathrm{P} 0$ of Trypanosoma cruzi and a functional epitope on the human $\beta 1$-adrenergic receptor. J. Exp. Med. 182:59-65.

15. Kalil, J., and Cunha-Neto, E. 1996. Autoimmunity in Chagas disease cardiomyopathy: fulfilling the criteria at last? Parasitol. Today. 12:396-399.

16. Masuda, M., et al. 1998. Functionally active cardiac antibodies in chronic Chagas' disease are specifically blocked by Trypanosoma cruzi antigens. FASEB J. 12:1551-1558.

17. Maeji, N.J., Bray, A.M., and Geysen, H.M. 1990. Multi-pin peptide synthesis strategy for $\mathrm{T}$ cell determinant analysis. J. Immunol. Methods. 134:23-33.

18. Harlow, E., and Lane, D. 1988. Antibodies: a laboratory manual. Cold Spring Harbor Laboratory Press. Cold Spring Harbor, New York, USA. 555-612.

19. Knoerzer, W., Binder, H., Schneider, K., Gruss, P., and McCarthy, J. 1989. Expression of synthetic genes encoding bovine and human basic fibroblast growth factors (bFGFs) in Escherichia coli. Gene. 75:21-30.

20. Schneppe, B., Eichner, W., and McCarthy, J. 1994. Translational regulation of a recombinant operon containing human platelet-derived growth factor (PDGF)-encoding genes in Escherichia coli: genetic titration of the peptide chains of the heterodimer AB. Gene. 143:201-209.

21. Brener, Z. 1962. Therapeutic activity and criterion of cures on mice experimentally infected with T. cruzi. Revista Instituto de Medicina Tropical de São Paulo. 4:389-439.

22. Alcina, A., and Fresno, M. 1987. Activation by synergism between endotoxin and lymphokines of the mouse macrophage cell line J774 against infection by Trypanosoma cruzi. Parasite Immunol. 9:175-186.

23. Heidehain, H.E. 1896. Noch einmal uberdie darstellung der central korpev durch eisenhamatoxylin nebst einígen allgemeinen bemerkungen uber die hamatoxylinfarben. Z. Wiss. Mikrosk. 13:186-189.

24. Van Gieson, J. 1889. Laboratory notes of technical methods for the nervous tissue stain (Hematoxylin, acid fuchsin and picric acid as nervous tissue stain). New York Med. J. 50:57-60.

25. Weise, M.J., and Carnegie, P.R. 1988. An approach to searching protein sequences for superfamily relationships or chance similarities relevant to the molecular mimicry hypothesis: application to the basic proteins of myelin. J. Neurochem. 51:1267-1273.

26. Abrahamsohn, I.A., and Coffman, R.L. 1995. Cytokine and nitric oxide regulation of the immunosuppression in Trypanosoma cruzi infection. J. Immunol. 155:3955-3963.

27. Rowland, E., Lozykowski, M., and McCormick, T. 1992. Differential cardiac histopathology in inbred mouse strains chronically infected with Trypanosoma cruzi.J. Parasitol. 78:1059-1066.

28. Schenkman, S., Eichinger, D., Pereira, M., and Nussenzweig, V. 1994. Structural and functional properties of Trypanosoma trans-sialidase. Annu. Rev. Microbiol. 48:499-523.

29. Frevert, U., Schenkman, S., and Nussenzweig, V. 1991. Stage-specific expression and intracellular shedding of the cell surface trans-sialidase of Trypanosoma cruzi. Infect. Immun. 60:2349-2360.

30. Damian, R.T. 1964. Molecular mimicry: antigen sharing by parasite and host and its consequences. Am. Nat. 98:129-149.

31. Cammer, W., Bloom, B., Norton, W., and Gordon, S. 1978. Degradation of basic protein in myelin by neutral proteases secreted by stimulated macrophages. Proc. Natl. Acad. Sci. USA. 75:1554-1558.

32. Tarleton, R.L., Zhang, L., and Downs, M.O. 1997. "Autoimmune rejection" of neonatal heart transplants in experimental Chagas disease is a parasite-specific response to infected host tissue. Proc. Natl. Acad. Sci. USA. 94:3932-3937. 